\title{
2-JY SAMPLE OF SOUTHERN RADIO SOURCES
}

\author{
R. MORGANTI ${ }^{1,2}$, C.N. TADHUNTER ${ }^{3}$, R. DICKSON ${ }^{3}$ \\ M. SHAW ${ }^{3}$, T.A. OOSTERLOO ${ }^{2}$, AND J.E.REYNOLDS ${ }^{2}$ \\ 1 Istituto di Radioastronomia, Bologna, Italy \\ 2 Australia Telescope National Facility, Epping, Australia \\ 3 Department of Physics, University of Sheffield, UK
}

We have collected multi-waveband (radio, optical and X-ray) data for a complete sample of southern radio sources. The sample includes 88 objects selected from the Wall \& Peacock (1985) catalogue that is complete down to $S_{2.7 G H z}=2 \mathrm{Jy}, \delta<10^{\circ}$ and the $z<0.7$. This database (Tadhunter et al. (1993), Morganti et al. (1993), and Siebert et al. these Proceedings) provides an important tool for investigating the nature of anisotropies and orientation effects in AGN and the physical causes of the correlation between their emission at different frequencies.

\section{Radio cores}

High-resolution observations of the radio cores have been done using the Parkes-Tidbinbilla real-time interferometer (PTI). We have measured the core flux densities at $2.3 \mathrm{GHz}$ with a resolution of $\sim 00^{\prime \prime} 1(\sim 1 \mathrm{kpc}$ at the redshifts of our sources, $H_{\circ}=50 \mathrm{~km} \mathrm{~s}^{-1} \mathrm{Mpc}^{-1}$ and $\left.q_{\circ}=0\right)$. The radiocore dominance derived using these data $\left(R=S_{\text {core }} / S_{\text {ext }}\right)$ shows different distributions for different optical characteristics of the radio galaxies (RG) (Fig. 1). Among the FR II radio galaxies, narrow-line RGs (NLRGs) show the lower value of $\log R$ while broad-line RGs (BLRGs) have the largest $\log R$. There is also a continuity in the distribution of $\log R$ going from the NLRGs to the steep and flat-spectrum quasars (SSQ and FSQ in Fig. 1). This result supports the idea that BLRGs are more beamed toward us (compared to other RGs) either because they are objects intermediate between NLRGs and quasars, or because they represent low- $z$ quasars. This result is also consistent with the higher detection rate and luminosity of 

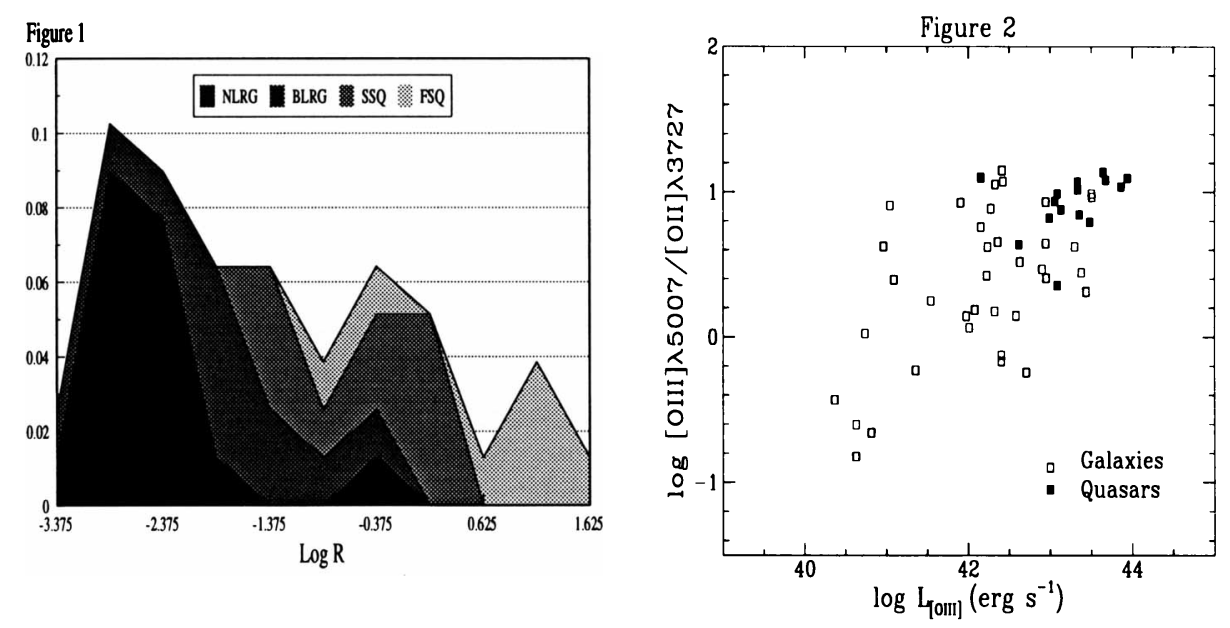

BLRGs in the soft X-rays compared to the other RGs (Siebert et al. these Proceedings).

\section{Optical properties}

Strong emission lines appear only if the total radio power is $\gtrsim 10^{26} \mathrm{~W} \mathrm{~Hz}^{-1}$. However, a significant number of galaxies with only absorption lines or emission lines of small equivalent width in their spectra have FRII radio sources. These objects can be identified with the "low excitation" objects highlighted by Laing (1994). A correlation between [O III] $]$ 5007/[O II] $] \lambda 3727$ v. $\log L_{[O I I I]}$ is observed at low $L_{[O I I I]}$ (Fig. 2) but the correlation flattens for high [O III] $\lambda 5007$ luminosity (and higher $z$ ). This can be explained if the gas properties or the balance between the ionization mechanisms (photoionization or shocks) change with $z$ or radio power.

Optical polarimetry is being carried out for the objects with $0.15<z<$ 0.7 in the $B$-band (corresponding to the rest-frame UV). Although some of the objects are highly polarized in the UV, for many we have failed to detect significant polarization. In general, the diversity of UV polarization properties we find in powerful radio galaxies at intermediate $z$ suggests that the UV excesses observed in these objects are unlikely to result solely from scattered light.

\section{References}

Laing, R.A. 1994, in ASP Conf.Ser. 54, p227;

Morganti, R., Killeen, N.E.B. \& Tadhunter, C.N. 1993, MNRAS, 263, 1023;

Tadhunter, C.N., Morganti, R., di Serego Alighieri, S., Fosbury, R.A.E. \& Danziger, I.J. 1993, MNRAS, 263, 999;

Wall, J.V. \& Peacock, J.A. 1985, MNRAS, 216, 173. 